auf das seinerzeit von W. Weber gegebene Gesetz für bewegte Elektricitätsmengen verwiesen.

Wassmuth.

\title{
La theorie de Maxwell et les oscillations hertziennes.,
} per H. Poincaré.

Le Magnétisme du Fer, per Ch. Mourain.

Eine Anzahl von französischen Gelehrten hat sich zusammengefunden, um eine gemeinverständliche Darstellung neverer Wissensgebiete, insbesonders der leitenden Ideen gewisser Theile der Physik und Biologie zu bringen. Diese Sammlung erscheint in einzelnen Heften (à 2 frcs.) unter dem Titel: "Scientia" und liegen einstweilen die obigen Hefte vor.

Das erste derselben bringt aus der kundigen Feder des Herrn Poincaré in knapper und doch anschaulicher Form eine Darstellung der theoretischen Grundgedanken Maxwell's und eine Schilderung der Hertzschen Schwingungen.

Nach einer allgemeinen Einleitung wird auf Maxwell's Theorie, insbesonders auf die der "Verschiebungsströme" eingegangen, der elektrischen Schwingungsversuche „vor Hertz", also jener von Feddersen und der zugehörigen Theorie Lord Kelvin's gedacht, woran sich naturgemäß eine Schilderung dex Untersuchungen von Hertz, des von ihm angewandten Erregers, Resonators, der Wärme- und mechanischen Vorgänge und der Fortpflanzung längs eines Drahtes (Hertz, Blondlot, Fizeau, Gounelle) anschliert. Es folgt die Behandlung stehender elektrischer Wellen und jener der vielfachen Resonanz, der Hinweis auf die Versuche von Garbasso, Zehnder, Strindberg, Perot, Jones und Decombe und die Ausbreitung der Wellen in der Luft nach den Versuchen von Hertz, Sarasin und de la Rive sowie die Fortpflanzung in dielektrischen Medien.

Nun werden sehr rasche Schwingungen also solche von kleiner Wellenlänge ins Auge gefasst und selbstredend an dieser Stelle des Apparates von Righi (mit den Kugeln in Öl) und des von Bose gedacht. Hiemit ist dann der Übergang gegeben zur Nachahmung optischer Erscheinungen d. i. der Herstellung elektrischer Schwingungen, welche die Vorgänge bei der Interferenz, Beugung, Polarisation durch Reflexion, Brechung, totalen Reflexion und Doppelbrechung wiedergeben.

Das zweite der obigen Hefte bringt eine von Herrn Mourain herrührende Darstellung der Lehren des Magnetismus. Die Stärke $H$ des magnetischen Feldes, die Intensität $J$ d. i. das magnetische Moment der Volumseinbeit, die magnetische Induction $B$ werden definiert, der Zusammenhang dieser Größen d. i. die Gleichung. $B=H+4 \pi J$ aufgestellt and die Begriffe: Magnetisierungszahl (Susceptibilität) $K=J: H$ und Durchlässigkeit (Permeabilität) $\mu=B: H$ erläutert. Es folgt eine Darstellung des allgemeinen Charakters der Magnetisierungscurven $(H, B$ und außerdem $H, K)$ und jener der Hyssteresis sowie die Schilderung einiger, zur Aufstellung solcher Curven nöthigen Versuche. So werden kurz besprochen: die magnetomotorischen und ballistischen Methoden, die von Hopkinson, die magnetische Wage von $\mathrm{Du}$ Bois u. a. - Es wird sowohl der Fall sehr schwacher Feldstärken wie auch der von nngemein großen, wo also Sättigung vorhanden ist, an der Hand neuerer Versuche dargestellt. Auf den. Einfluss der Form des zu magnetisierenden Körpers resp. auf das mögliche Auftreten entmagnetisierender Kräfte - der sogenannten freien 